\title{
Relaciones entre Estilos Educativos Parentales y Agresividad en Adolescentes
}

\author{
De la Torre-Cruz, M. J., García-Linares, M. C. y \\ Casanova-Arias, P.F.
}

Departamento de Psicología, Universidad de Jaén, Jaén

\section{España}

Correspondencia: Manuel J. de la Torre Cruz. Departamento de Psicología, Edif. C-5. Las Lagunillas s/n. Jaén. España. E-mail: majecruz@ujaen.es

(C) Education \& Psychology I+D+i and Ilustre Colegio Oficial de Psicología de Andalucia Oriental (Spain) 


\section{Resumen}

Introducción. La agresividad física y verbal que niños y adolescentes dirigen hacia sus iguales se relaciona con las pautas de actuación o los estilos educativos que emplean sus progenitores. El objetivo de nuestra investigación residió en examinar la relación existente entre la percepción que un grupo de jóvenes adolescentes tenía del estilo educativo exhibido por sus progenitores (madres y padres) y el nivel de agresividad física, verbal, ira y hostilidad que manifestaban hacia sus iguales.

Método. 371 estudiantes con edades comprendidas entre los 12 y los 16 años emitieron sus respuestas a varias medidas de autoinforme: la Escala de Afecto (EA) y la Escala de Normas y Exigencias (ENE) así como, al Cuestionario de Agresividad.

Resultados. El análisis de varianza reveló que los adolescentes que atribuían a madres y padres un estilo de socialización democrático obtenían menores puntuaciones en las dimensiones agresividad física y verbal que sus iguales que etiquetaron a sus madres y padres como autoritarios. Adicionalmente, los chicos informaron ser físicamente más agresivos que las chicas.

Discusión y Conclusión. La percepción de un estilo parental autoritario parece asociada al incremento de las manifestaciones de agresividad. La percepción de un bajo nivel de afecto y un elevado grado de control, propia de un estilo autoritario, se vincula con la expresión de conductas agresivas que podrían deteriorar seriamente el ajuste que los adolescentes exhiben en diferentes contextos como el de las interacciones con los iguales.

Palabras Clave: estilos educativos, adolescentes, agresividad física y verbal. 


\title{
Relations between Childraising Styles and Aggressiveness in Adolescents
}

\begin{abstract}
Introduction. Physical and aggressive behavior which children and adolescents show toward peers is associated to parenting styles. The aim of this research was to examine the relation between perceived parenting styles (from mothers and fathers) and the level of physical and verbal aggressive behavior, anger and hostility showed towards the peers.
\end{abstract}

Method. Thirty hundred and seventy-one students, aged $12-16$ years old, answered to several self-report measures: Escala de Afecto (EA) and Escala de Normas y Exigencias (ENE) as well as, Aggressive Questionnaire.

Results. Analysis of variance revealed that adolescents whose parents showed an authoritative parenting style obtained lower scores in physical and verbal aggressive behaviors than peers whose parents showed an authoritarian style. Moreover, boys scored higher than girls in physical aggressive behavior.

Discussion and Conclusion. A perceived authoritarian parenting style was associated with a higher aggressive behavior. Low levels of affectio and high levels of control, which define an authoritarian style, were associated with higher levels of aggressive behavior, which could negatively affect the behavior exhibited by adolescents in different context as peer interactions.

Keywords: parenting styles, adolescents, physical and verbal aggressive behavior. 


\section{Introducción}

Las interacciones que tienen lugar en el ambiente familiar desempeñan un importante papel en los procesos de aprendizaje de niños y adolescentes al favorecer la adquisición de conductas sociales que se generalizan a otros contextos, como el de las relaciones con los iguales (Lamborn y Felbab, 2003; Lamborn, Mounts, Steinberg y Dornbusch, 1991). Asimismo, diferentes tipos de prácticas educativas familiares, tales como el afecto, la receptividad, la supervisión o la disciplina se relacionan con importantes indicadores de desarrollo tales como el bienestar físico y emocional, la vulnerabilidad al estrés, el logro académico, la conducta altruista o el comportamiento agresivo y antisocial que los hijos exhiben hacia sus compañeros (De la Torre, Casanova, García, Carpio y Cerezo, 2011; Hernando, Oliva y Pertegal, 2012; Kim, Brody y Murry, 2003; Oliva, Parra y Arranz, 2008; Steinberg, Lamborn, Darling, Mounts y Dornbusch, 1994; Tur-Porcar, Mestre, Samper y Malonda, 2012).

Dos de estas prácticas han centrado la mayor parte de la investigación que examina las relaciones entre padres e hijos: el afecto y el control. La consideración conjunta de las dimensiones afecto y control ha posibilitado la configuración de los diferentes estilos educativos parentales. De acuerdo con Glasgow, Dornbusch, Troyer, Steinberg y Ritter (1997), los estilos educativos son un compendio de actitudes, conductas y expresiones no verbales que caracterizan la naturaleza de las relaciones mantenidas entre padres e hijos en diferentes escenarios. La combinación de altos y bajos niveles de afecto y control posibilita la configuración de cuatro estilos educativos: democrático, permisivo, autoritario y negligente (Baumrind, 1971; Maccoby y Martin, 1983).

El estilo educativo democrático se caracteriza por equilibrar eficazmente elevados niveles de afecto y control. Los progenitores incluidos en esta categoría supervisan el comportamiento de sus hijos y apelan a su autoridad como padres cuando desobedecen las normas. El estilo negligente se define por la ausencia de control y despreocupación por la conducta e intereses de los hijos. Un patrón permisivo se fundamenta en niveles elevados de afecto y ausencia de control que se traducen en un grado insuficiente de exigencias de madurez. Finalmente, un estilo autoritario se concreta por la escasez de muestras de afecto y el interés por el control tanto de las conductas como de las actitudes de niños y adolescentes, enfatizando la obediencia sin discusión y el respeto a la autoridad paterna. 
Dado que las figuras parentales son la primera y principal fuente de socialización de los hijos, al tiempo que un modelo para el establecimiento de relaciones con los iguales y promotores de bienestar, distintas investigaciones han centrado su atención en conocer la posible incidencia de tales prácticas o estilos sobre la manifestación de la conducta agresiva, en su forma física, verbal o relacional, de niños y adolescentes (Buschgens, van Aken, Swinkels, Ormel, Verhulst y Buitelaar, 2010; Côté, Vaillancourt, Barker, Nagin y Tremblay, 2007; Kawabata, Alink, Tseng, van Ijzendoorn y Crick, 2011; Underwood, Beron y Rosen, 2009; Vaillancourt, Miller, Fagbemi, Côté y Tremblay, 2007).

Algunas estudios evidencian la existencia de relaciones entre prácticas educativas parentales y comportamientos agresivos en niños y adolescentes. Buschgens et al. (2010), plantearon si la percepción de diversos tipos de prácticas parentales incrementaba el riesgo de conductas externalizantes en una muestra de preadolescentes. Los resultados obtenidos mostraron que los hijos que atribuían a sus progenitores una falta de apoyo emocional así como elevados niveles de rechazo y sobreprotección fueron considerados por padres y profesores como jóvenes más agresivos que exhibían, al tiempo, comportamientos delictivos. Kawabata et al. (2011), emplearon un procedimiento de metaanálisis con el objetivo de analizar e integrar los hallazgos existentes relativos a la asociación entre diferentes patrones de actuación parental (práctica educativa positiva, control psicológico, imposición disciplinar severa e indulgencia) y el grado de agresión relacional expresado por niños y adolescentes. Los resultados revelaron que los niveles de agresión relacional disminuían a medida que incrementaban las conductas ligadas a una paternidad positiva. Asimismo, la percepción de una mayor rigidez disciplinar e indulgencia materna así como el ejercicio de un elevado control psicológico paterno se asoció con índices más elevados de agresividad relacional infantil y juvenil.

En una lína similar, Côté et al., (2007) trataron de identificar en su estudio diferentes variables familiares que podrían predecir las trayectorias evolutivas conjuntas de agresividad física y social expresadas durante la niñez media. Entre otras actuaciones, un comportamiento materno hostil (enfados, desaprobación constante o incapacidad para controlar la conducta de su hijo) cuando los pequeños tenían dos años de edad predecía los niveles elevados y atípicos de agresividad física y relacional exhibidos a los ocho años de edad. Más recientemente, Underwood et al., (2009), condujeron un estudio que tenía como objetivo fundamental examinar el desarrollo de la agresión física y social en chicos y chicas de nueve a 13 años de edad así como conocer algunos de los predictores familiares que podrían dar cuenta de las diferentes 
trayectorias de conducta agresiva manifestada. Los resultados obtenidos revelaron que el desempeño de un estilo educativo familiar tanto permisivo como autoritario predecía la inclusión de varios de estos preadolescentes en un grupo particular caracterizado por mostrar una trayectoria ascendente en la manifestación tanto de agresividad física como social evaluada por sus profesores.

Atendiendo a un contexto nacional, Estévez, Musitu y Herrero (2005), exploraron entre otras cuestiones el papel que los padres desempeñaban en la relación existente entre conducta agresiva y ajuste psicosocial. Para ello, una muestra de adolescentes con edades comprendidas entre los 11 y 16 años de edad informó de la frecuencia con la que habían manifestado conductas agresivas, físicas y verbales, hacia sus iguales a lo largo del último año. Adicionalmente, informaron sobre la facilidad y dificultad existente en la comunicación familiar mantenida con padres y madres. Los resultados obtenidos revelaron que la existencia de una comunicación abierta con ambos progenitores se relacionaba de forma negativa con la cantidad de comportamientos agresivos exhibidos mientras que el mal entendimiento o falta de comunicación entre padres e hijos se asoció con una mayor frecuencia informada de conductas agresivas. Asimismo, Gallarin y Alonso-Arbiol (2012), hallaron relaciones negativas entre implicación parental y agresividad así como positivas entre imposición y agresividad en una muestra de adolescentes españoles. Tur-Porcar et al., (2012), establecieron como objetivo analizar el papel de un conjunto de variables de crianza negativa (control, permisividad, hostilidad y negligencia) sobre la agresividad física y verbal de los hijos, atendiendo a las posibles diferencias en función del progenitor. Los resultados mostraron que prácticas tales como la hostilidad y permisividad mantenían relación con la agresividad expresada por los niños y adolescentes. Concretamente, la hostilidad materna predecía la agresión exhibida por chicos y chicas, mientras que la permisividad se relacionaba de forma diferencial según el sexo del hijo. Las chicas se mostraban más agresivas ante el exceso de permisividad paterna, mientras que lo contrario sucedía en el caso de los chicos.

De los anteriores estudios parece constatarse que la agresividad física, verbal o relacional que niños y adolescentes dirigen hacia sus iguales se relaciona con las pautas de actuación o los estilos educativos que emplean sus progenitores. La investigación precedente sugiere que los jóvenes educados en hogares caracterizados por la ausencia de afecto, el escaso intercambio comunicativo o la imposición severa de normas, expresiones acordes al ejercicio de un estilo de socialización parental autoritario, muestran una mayor frecuencia de compor- 
tamientos agresivos hacia sus iguales (Côté et al., 2007; Estévez et al., 2005; Hale III, van der Valk, Engels y Meeus, 2005; Kawabata et al., 2011; Tur-Porcar et al., 2012).

\section{Objetivos e hipótesis}

Así pues, el objetivo de nuestra investigación residió en examinar la relación existente entre la percepción que un grupo de jóvenes adolescentes tenía del estilo educativo exhibido por sus progenitores (madres y padres) y el nivel de agresividad física, verbal, ira y hostilidad que manifestaban hacia sus iguales. La consideración por separado de la percepción mantenida sobre madres y padres es una aportación de este trabajo debido a la cada vez mayor importancia concedida a la figura del padre en la crianza y socialización de los hijos (Hoeve, Dubas, Gerris, van der Laan y Smeenk, 2011; McKinney y Renk, 2008; Rodríguez, Peña y Torío, 2009). Buena parte de la investigación precedente establece un estilo de socialización familiar, promediando la puntuación alcanzada por madres y padres, sin considerar la relación específica que el estilo educativo exhibido por cada progenitor pueda tener sobre el ajuste psicosocial de sus hijos adolescentes.

Asimismo, la práctica totalidad de estudios que analizan los estilos educativos parentales ha utilizado dos dimensiones, afecto y control, para configurar las cuatro categorías tradicionales. Sin embargo, parece razonable plantear que las formas de control que exhiben los padres democráticos difieran notoriamente de aquellas que utilizan los padres autoritarios aún cuando es cierto que para la conformación de los cuatro estilos clásicos la dimensión control considerada haya sido solo una. En la caracterización de los diferentes estilos diversos autores (Baumrind, 1991; Chao, 2001, Dwairy, 2008; Glasgow et al., 1997; Steinberg, 2001), coinciden en señalar que los padres democráticos son figuras de autoridad que han de ser respetadas, pero, al tiempo se muestran sensibles a las necesidades de los hijos, mientras que los autoritarios se autoproclaman a sí mismos como figuras de autoridad que apelan a la obediencia por la obediencia. Asimismo, los padres democráticos establecen límites y normas sobre el comportamiento que esperan en sus hijos, normas que son explicadas y razonadas y que incluso pueden llegar a debatirse o discutirse respetuosamente. Por el contrario, los padres autoritarios justifican la existencia de las normas apelando al "porque sí" o "porque yo lo he dicho". La regla es incuestionable y de su incumplimiento puede derivarse la imposición de castigos, hecho que denota una gran rigidez e inflexibilidad. Por ello, en este estudio se emplearon conjuntamente tres dimensiones (afecto, disciplina/control inductivo y disciplina/control rígido) en un intento de delimitar con mayor precisión los distintos estilos de socia- 
lización familiar, dado que entendemos que los progenitores democráticos y autoritarios no solo difieren entre sí en el grado de afecto que expresan sino, además, en el modo preferente de control que exhiben hacia sus hijos (más flexible en los primeros y más severo en los segundos).

Así pues, se hipotetizó que aquellos chicos y chicas que perciben en sus progenitores el ejercicio de una maternidad y paternidad tanto autoritaria como negligente expresarán una mayor agresividad hacia sus iguales. Adicionalmente se planteó la existencia de diferencias en la manifestación de agresividad en función del sexo del adolescente. Estudios previos han revelado que los chicos se implican con mayor frecuencia en formas físicas de agresión, mientras que las chicas exhiben un número más elevado de acciones agresivas de carácter relacional (Bjorkqvist, Lagerspetz y Kaukiainen, 1992; Toldos, 2005). Según Kistner, Counts-Allan, Dunke, Drew, David-Ferdon y Lopez (2010) estas diferencias en el patrón de conducta agresiva en función del sexo se hacen más evidentes al inicio de la educación secundaria. Consiguientemente, se predijo que los chicos obtendrían mayores puntuaciones que las chicas en la manifestación de agresividad física, mientras que serían las chicas las que alcanzarían valores más elevados en agresividad verbal.

\section{Método}

\section{Participantes}

Inicialmente, 886 estudiantes con edades comprendidas entre los 12 y los 16 años $(M$ $=13.07, S D=.97)$, de primer $(\mathrm{n}=483,54.5 \%)$ y segundo curso $(\mathrm{n}=403,45.5 \%)$ de educación secundaria obligatoria (46 aulas implicadas), pertenecientes a siete centros educativos (cinco públicos y dos concertados), de dos ciudades de la Comunidad Autónoma de Andalucía participaron en este estudio. Cuatro centros estaban situados en la zona centro de las ciudades, mientras que los otros tres se ubicaban en la periferia. La distribución en función de la variable sexo fue de 449 chicos (50.7\%) y 437 chicas (49.3\%). El $97.2 \%$ era de nacionalidad española. La muestra fue incidental y estuvo en función de la disponibilidad y accesibilidad de los centros educativos. De todos ellos, los datos de 371 alumnos (173 chicos, $46.6 \%$ y 198 chicas, 53.4\%), aquellos cuyas puntuaciones asignadas posibilitaron la clasificación de ambos progenitores en cualquiera de los estilos de socialización considerados, fueron empleados en posteriores análisis. El promedio de edad de este grupo reducido fue similar al obtenido para 
la totalidad de participantes $(M=13.10, S D=1.00)$. La distribución atendiendo al curso fue de 187 estudiantes de primer (50.45\%) y 184 (49.6\%) de segundo curso.

\section{Instrumentos}

Escala de Afecto (EA) y Escala de Normas y Exigencias (ENE) (Fuentes, Motrico y Bersabé, 1999; Bersabé, Fuentes y Motrico, 2001).

Con objeto de establecer los estilos educativos maternos y paternos se emplearon los ítems de la dimensión "afecto y comunicación" (p.e.: "Dedica tiempo a hablar conmigo") de la Escala de Afecto (EA), así como los ítems que conforman las dimensiones "disciplina inductiva" (p.e.: “Antes de castigarme escucha mis razones") y "disciplina rígida” (p.e.: "Me exige que cumpla las normas aunque no las entienda") de la versión para hijos de la Escala de Normas y Exigencias (ENE) elaboradas por Bersabé et al., (2001). Todas las dimensiones citadas se componen de 10 ítems con un formato de respuesta tipo Likert a lo largo de una escala de cinco puntos $(1=$ nunca, $2=$ pocas veces, $3=$ algunas veces, $4=$ a menudo y $5=$ siempre) que evalúa la frecuencia con la que los adolescentes perciben ciertas actuaciones en sus progenitores. Una elevada puntuación equivale a una mayor percepción de afecto así como de disciplina rígida e inductiva. Los índices de fiabilidad obtenidos en este estudio mediante el coeficiente alpha de Cronbach para madres y padres, en este orden, fueron: afecto (.87 y .89), disciplina inductiva (.85 y .86), disciplina rígida (.74 y .74).

En el estudio original que analizó las propiedades psicométricas de la Escala de Afecto (EA) y la Escala de Normas y Exigencias (ENE), el índice de consistencia interna osciló desde .72 (disciplina rígida materna) hasta .90 (afecto y comunicación paterna). Asimismo, las correlaciones entre las medidas de estos constructos (afecto y comunicación, disciplina inductiva y disciplina rígida) empleando métodos diferentes fueron elevadas. Concretamente, la correlación entre la dimensión afecto y comunicación de la Escala de Afecto y la medida de afecto del Inventory of Parent and Peer Attachment, "IPPA" (Armsden y Greenberg, 1987) fue superior a .70, mientras que el valor de las correlaciones entre las dimensiones disciplina inductiva y rígida y dos de los tres prototipos (democrático y autoritario) de autoridad parental empleando el Parental Authority Questionnaire "PAQ" (Buri, 1991) fueron superiores a .49 (Bersabé et al., 2001). 
Aggressive Questionnaire (AQ, Buss y Perry, 1992) Cuestionario de Agresividad (Andreu, Peña y Graña, 2002).

El cuestionario de agresividad es uno de los instrumentos más utilizados en la evaluación del comportamiento agresivo en la edad adolescente (García-León, Reyes, Vila, Pérez, Robles y Ramos, 2002; Morales-Vives, Codorniu-Raga, Vigil-Colet, 2005; Santisteban y Alvarado, 2009). En este estudio se empleó la versión de 26 ítems propuesta por Andreu et al., (2002). Los autores hallaron una estructural factorial de cuatro dimensiones: agresividad física, ocho ítems (p.e.: “A veces no puedo controlar el impulso de golpear a otra persona”), agresividad verbal, cinco ítems (p.e.: "Cuando no estoy de acuerdo con mis amigos/as, discuto abiertamente con ellos/as"), ira, 6 ítems (p.e.: "Cuando estoy frustrado/a muestro el enfado que tengo") y hostilidad, 7 ítems (p.e.: “A veces soy bastante envidioso/a”) que dan cuenta de los aspectos cognitivos (pensamientos negativos, resentimiento, desconfianza), comportamentales (agresión física y verbal) y afectivo-emocionales (rabia, cólera o repulsa) de la agresividad. El cuestionario presenta un formato tipo Likert con cinco opciones de respuesta donde se indica en qué medida el contenido del ítem se adecua al comportamiento exhibido por el encuestado $(1=$ completamente falso, $\ldots, 5=$ completamente verdadero $)$. Una elevada puntuación se corresponde con un mayor comportamiento agresivo y hostil. Los índices de fiabilidad calculados mediante el coeficiente alpha de Cronbach fueron: .86 para agresividad física, .71 para agresividad verbal, .67 para ira y .76 para hostilidad. Estos valores son muy similares a los hallados por Andreu et al. (2002). En su estudio de validación del cuestionario con una muestra de adolescentes y jóvenes españoles los índices de fiabilidad oscilaron desde .68 para la dimensión agresividad verbal hasta .86 para la dimensión agresividad física. Además, la realización de un análisis factorial confirmatorio reveló la presencia de una estructura tetrafactorial que se corresponde con cada una de las subescalas previamente descritas.

\section{Procedimiento}

Con objeto de acceder a la muestra de interés tuvo lugar un encuentro con los miembros del equipo directivo de los centros educativos a quienes se comunicó los objetivos del estudio y se solicitaba la aprobación para la aplicación de pruebas. Adicionalmente, se remitieron cartas a los progenitores por medio tanto de las asociaciones de madres y padres de alumnos como de sus propios hijos, en las que se describía el propósito de investigación y se solicitaba la conformidad de participación de los últimos. Se utilizó un procedimiento de consentimiento pasivo (informados madres y padres tenían la posibilidad de devolver la carta 
firmada si no estimaban conveniente la participación de sus respectivos hijos e hijas). Ochenta familias firmaron el documento adjunto a la carta informativa declinando la participación de su respectivo hijo o hija en el estudio. Las distintas pruebas se aplicaron en el horario habitual de clase. Ningún alumno se ausentó del aula durante la aplicación de las pruebas y quienes no fueron autorizados dedicaron el tiempo requerido a la realización de tareas académicas.

Para establecer los estilos maternos y paternos se consideraron simultáneamente las puntuaciones obtenidas en las tres dimensiones mencionadas (afecto, disciplina inductiva y disciplina rígida). Los valores de la mediana en estas dimensiones cuando el juicio del adolescente se refería a la madre fueron 42,40 y 29 , respectivamente, mientras que cuando el juicio se circunscribía al padre fueron de 38, 40 y 28. Atendiendo a estos valores, los adolescentes que asignaron a cualquiera de sus progenitores puntuaciones inferiores a la mediana en las diferentes dimensiones se incluyeron en el grupo de progenitores percibidos como negligentes mientras que, aquellos que concedieron puntuaciones superiores a la mediana en las dimensiones afecto y disciplina inductiva e inferiores a la mediana en disciplina rígida se incluyeron en el grupo de progenitores democráticos. Adicionalmente, los jóvenes que asignaron puntuaciones inferiores a la mediana tanto en afecto como en disciplina inductiva, pero, superiores a su respectiva mediana en disciplina rígida fueron incluidos en el grupo de progenitores percibidos como autoritarios. Por último, quienes asignaron puntuaciones superiores a la mediana en afecto e inferiores a la mediana en ambas formas disciplinarias conformaron el grupo de progenitores permisivos. Se excluyeron de posteriores análisis aquellos casos en los que o bien el valor en alguna de las dimensiones era igual a su respectiva mediana, o bien, la combinación de puntuaciones no se correspondía a cualquiera de los patrones descritos.

Conforme a este criterio de clasificación, 146 madres (39.4\%) fueron percibidas como negligentes, $102(27.5 \%)$ democráticas, 80 (21.6\%) autoritarias y, finalmente, 43 (11.6\%), permisivas. Cuando esta clasificación se circunscribió a los padres la frecuencia de cada una de las categorías fue de 132 (35.6\%), 102 (27.5\%), 91 (24.5\%) y 46 (12.4\%), respectivamente. Estos porcentajes son muy similares a los obtenidos por Glasgow et al., (1997) en su estudio con adolescentes norteamericanos empleando en su clasificación las dos dimensiones tradicionales, afecto y control.

El procedimiento descrito supone una clasificación más rigurosa del estilo de socialización familiar, aun a riesgo de reducirse el tamaño muestral, al considerar conjuntamente dos 
tipos de actuaciones disciplinarias o formas de control diferentes, una más razonada que promueve una mayor autonomía y participación del adolescente en la toma de decisiones (disciplina inductiva), propia de una actuación parental más democrática y, otra más impositiva, taxativa y prohibitiva (disciplina rígida), acorde con la expresión de un estilo educativo más autoritario (Buri, 1991).

\section{Análisis Estadístico}

Los análisis estadísticos empleados fueron análisis multivariados de varianza (MANOVA), el análisis univariado de varianza (ANOVA) y comparaciones post-hoc en las que se empleó la prueba de Bonferroni.

\section{Resultados}

Como muestra la Tabla 1, se obtuvieron correlaciones estadísticamente significativas entre la práctica totalidad de dimensiones educativas consideradas y las medidas obtenidas en el cuestionario de agresividad. El patrón de resultados fue muy similar cuando el juicio del adolescente se refería tanto a la madre como al padre. Así, la percepción de un mayor afecto se relacionó negativamente con los niveles de agresividad física, verbal, ira y hostilidad, mientras que un mayor grado de disciplina rígida mantuvo relaciones positivas con todas las dimensiones evaluadas. Adicionalmente, la percepción de una disciplina inductiva tanto en padres como en madres se asoció negativamente con la agresividad física manifestada, mientras que solo la disciplina inductiva paterna mantuvo una relación negativa con la hostilidad.

Se realizaron dos análisis multivariados de varianza (MANOVA), uno para cada progenitor. Las variables independientes fueron el estilo de socialización atribuido y el sexo del adolescente. Las variables dependientes fueron las puntuaciones obtenidas en las dimensiones agresividad física, verbal, ira y hostilidad. El primer análisis reveló la existencia de un efecto principal estadísticamente significativo de la variable estilo materno percibido, $\lambda=.86$, $F(12,952.76)=4.68, p<.001, \eta^{2}=.05$, así como un efecto principal de la variable sexo, $\lambda=$ $.89, F(4,360)=10.35, p<.001, \eta^{2}=.10$. La interacción estilo materno percibido $\mathrm{x}$ sexo no resultó ser estadísticamente significativa, $\lambda=.96, F(12,952.76)=1.26, p>.05$. Análisis univariados posteriores mostraron la existencia de diferencias estadísticamente significativas en todas y cada una de las dimensiones evaluadas atendiendo al estilo materno percibido, $F(3$, $363)=14.20, p<.000, \eta^{2}=.11$ para la dimensión agresividad física, $F(3,363)=7.35$, < $.000, \eta^{2}=.06$, para la dimensión agresividad verbal, $F(3,458)=8.69, p<.001, \eta^{2}=.07$ para 
la dimensión ira y, finalmente, $F(3,458)=8.32, p<.05, \eta^{2}=.06$ en la dimensión hostilidad. Respecto al sexo únicamente se obtuvo una diferencia estadísticamente significativa en el grado de agresividad física manifestado, $F(1,363)=26.97, p<.001, \eta^{2}=.07$, siendo mayor en el caso de los chicos que de las chicas.

Tabla 1. Correlaciones bivariadas entre las diferentes dimensiones de socialización (maternas y paternas) y las medidas de agresividad física, verbal, ira y hostilidad.

\begin{tabular}{|c|c|c|c|c|c|c|c|c|c|c|}
\hline & 1 & 2 & 3 & 4 & 5 & 6 & 7 & 8 & 9 & 10 \\
\hline Afecto (M) & 1 & $.75^{* *}$ & $-.28 * *$ & $.66^{* *}$ & $.59 * *$ & $-.19 * *$ & $-.30 * *$ & $-.12 * *$ & $-.17 * *$ & $-.21 * *$ \\
\hline Inductiva (M) & & 1 & $\begin{array}{r}- \\
.12 *\end{array}$ & $.60 * *$ & $.80 * *$ & -.02 & $-.21 * *$ & -.05 & -.10 & -.09 \\
\hline Rígida (M) & & & 1 & $\begin{array}{c}- \\
.23 * *\end{array}$ & -.09 & $.76^{* *}$ & $.27 * *$ & $.26 * *$ & $.32 * *$ & $.30 * *$ \\
\hline Afecto (P) & & & & 1 & $.80 * *$ & $-.28 * *$ & $-.27 * *$ & $-.20 * *$ & $-.21 * *$ & $\begin{array}{c}- \\
.22 * *\end{array}$ \\
\hline Inductiva (P) & & & & & 1 & $-.13 * *$ & $-.22 * *$ & -.10 & $\begin{array}{r}- \\
.13^{*}\end{array}$ & -.09 \\
\hline Rígida (P) & & & & & & 1 & $.28 * *$ & $.24 * *$ & $.27 * *$ & $.29 * *$ \\
\hline A. Física & & & & & & & 1 & $.52 * *$ & $.48 * *$ & $.42 * *$ \\
\hline A. Verbal & & & & & & & & 1 & $.54 * *$ & $.44 * *$ \\
\hline Ira & & & & & & & & & 1 & $.55 * *$ \\
\hline Hostilidad & & & & & & & & & & 1 \\
\hline
\end{tabular}

Comparaciones a posteriori empleando la prueba de Bonferroni revelaron la existencia de diferencias estadísticamente significativas en la dimensión agresividad física. Los jóvenes que atribuyeron a sus madres un estilo de socialización democrático manifestaron ser físicamente menos agresivos que sus iguales que atribuyeron a sus madres un estilo educativo tanto negligente como autoritario. Adicionalmente, los adolescentes cuyas madres ejercían un estilo negligente informaron de menores niveles de agresividad física que aquellos otros que percibían a sus madres como autoritarias $(M=14.70, M=18.15$ y $M=20.67)$. Con respecto a la dimensión agresividad verbal se hallaron diferencias entre los jóvenes que percibieron a sus madres como democráticas frente a quienes las consideraron como autoritarias, obteniendo los primeros una menor puntuación que los segundos en esta dimensión $(M=10.52$ y $M=$ 13.0). Asimismo, los niveles de ira fueron mayores, en comparación con el resto de encuestados, en aquellos adolescentes que atribuyeron a sus madres un estilo de socialización autoritario $(M=13.97, M=14.58, M=15.21$ y $M=17.34)$. Por último, la percepción de una madre 
autoritaria se asoció con un mayor índice de hostilidad en comparación con el obtenido cuando a éstas se atribuía un estilo tanto democrático como permisivo $(M=14.58, M=13.66 \mathrm{y}$ $M=17.98)$.

Tabla 2. Puntuaciones promedio y desviaciones típicas (entre paréntesis) en las medidas de agresividad física, verbal, ira y hostilidad en función del estilo de socialización materno.

Estilos de socialización maternos

\begin{tabular}{lcccccccc} 
& \multicolumn{2}{c}{ Democrático } & \multicolumn{2}{c}{ Permisivo } & \multicolumn{2}{c}{ Negligente } & \multicolumn{2}{c}{ Autoritario } \\
\cline { 2 - 9 } & Chica & Chico & Chica & Chico & Chica & Chico & Chica & Chico \\
& $(\mathrm{n}=63)$ & $(\mathrm{n}=39)$ & $(\mathrm{n}=28)$ & $(\mathrm{n}=15)$ & $(\mathrm{n}=72)$ & $(\mathrm{n}=74)$ & $(\mathrm{n}=35)$ & $(\mathrm{n}=45)$ \\
\hline A. Física & 13.59 & 15.82 & 13.04 & 19.53 & 17.00 & 19.30 & 18.54 & 22.80 \\
& $(5.66)$ & $(5.48)$ & $(5.14)$ & $(7.61)$ & $(6.89)$ & $(5.87)$ & $(7.55)$ & $(6.19)$ \\
A. Verbal & 10.94 & 10.10 & 10.25 & 12.87 & 11.63 & 12.14 & 13.17 & 12.84 \\
& $(3.60)$ & $(3.17)$ & $(3.42)$ & $(4.27)$ & $(3.34)$ & $(3.66)$ & $(3.78)$ & $(3.40)$ \\
Ira & 14.16 & 13.79 & 13.89 & 15.27 & 15.50 & 14.92 & 17.89 & 16.80 \\
& $(4.01)$ & $(4.48)$ & $(4.31)$ & $(4.64)$ & $(4.42)$ & $(4.28)$ & $(5.03)$ & $(4.94)$ \\
Hostilidad & 14.76 & 14.41 & 14.00 & 13.33 & 16.21 & 15.93 & 18.63 & 17.33 \\
& $(5.53)$ & $(5.47)$ & $(5.49)$ & $(4.40)$ & $(5.30)$ & $(5.009$ & $(4.99)$ & $(5.78)$ \\
\hline
\end{tabular}

El segundo de los análisis reveló la existencia de un efecto principal estadísticamente significativo de la variable estilo paterno percibido, $\lambda=.85, F(12,952.76)=5.13, p<$ $.001, \eta^{2}=.05$, así como de la variable sexo del adolescente, $\lambda=.90, F(4,363)=9.73, p<$ $.001, \eta^{2}=.10$. La interacción estilo paterno percibido $x$ sexo no resultó ser estadísticamente significativa, $\lambda=.97, F(12,952.76)=.81, p>.05$. Análisis univariados posteriores mostraron la existencia de diferencias estadísticamente significativas en todas y cada una de las dimensiones evaluadas, $F(3,363)=16.68, p<.001, \eta^{2}=.12$, para agresividad física, $F(3,363)=$ $7.22, p<.000, \eta^{2}=.06$, para agresividad verbal, $F(3,363)=10.25, p<.001, \eta^{2}=.08$ para ira y, finalmente, $F(3,363)=8.61, p<.001, \eta^{2}=.07$, en hostilidad. Respecto al sexo, se obtuvo una diferencia estadísticamente significativa en la dimensión agresividad física, $F(1,363)=$ $26.55, p<.000, \eta^{2}=.07$, siendo mayor en el caso de los chicos que de las chicas.

Comparaciones a posteriori empleando la prueba de Bonferroni arrojaron la existencia de diferencias estadísticamente significativas en la dimensión agresividad física entre 
los adolescentes que percibieron a sus padres como democráticos y permisivos frente a quienes lo hicieron como negligentes y autoritarios, mostrando los dos primeros menores índices de agresividad física que los dos restantes $(M=15.01, M=14.76, M=18.38$ y $M=20.41)$. Adicionalmente, se hallaron diferencias estadísticamente significativas en la dimensión agresividad verbal. Los hijos que percibían en sus padres el ejercicio de un estilo de socialización autoritario obtuvieron una mayor puntuación en esta dimensión que quienes atribuyeron a sus padres un estilo democrático $(M=10.71$ y $M=13.07)$. Finalmente, se obtuvieron diferencias estadísticamente significativas entre los jóvenes que atribuyeron a sus padres un estilo autoritario frente al resto en las dimensiones ira $(M=14.13, M=13.74, M=15.23$ y $M=17.32)$ y hostilidad $(M=14.66, M=14.10, M=15.70$ y $M=18.10)$. En cualquier caso, los hijos de padres autoritarios alcanzaron puntuaciones más elevadas en estas medidas.

Tabla 3. Puntuaciones promedio y desviaciones típicas (entre paréntesis) en las medidas de agresividad física, verbal, ira y hostilidad en función del estilo de socialización paterno.

\begin{tabular}{lcccccccc} 
& \multicolumn{7}{c}{ Estilos de socialización paternos } \\
\cline { 2 - 9 } & \multicolumn{2}{c}{ Democrático } & \multicolumn{2}{c}{ Permisivo } & \multicolumn{2}{c}{ Negligente } & \multicolumn{2}{c}{ Autoritario } \\
\cline { 2 - 9 } & Chica & Chico & Chica & Chico & Chica & Chico & Chica & Chico \\
& $(\mathrm{n}=62)$ & $(\mathrm{n}=40)$ & $(\mathrm{n}=27)$ & $(\mathrm{n}=19)$ & $(\mathrm{n}=71)$ & $(\mathrm{n}=61)$ & $(\mathrm{n}=38)$ & $(\mathrm{n}=53)$ \\
\hline A. Física & 13.73 & 16.30 & 11.41 & 18.11 & 16.70 & 20.05 & 19.71 & 21.60 \\
& $(5.89)$ & $(5.79)$ & $(3.19)$ & $(6.86)$ & $(6.20)$ & $(6.52)$ & $(8.04)$ & $(5.84)$ \\
A. Verbal & 10.68 & 10.73 & 10.04 & 12.53 & 11.72 & 11.82 & 13.39 & 12.74 \\
& $(3.55)$ & $(3.58)$ & $(2.31)$ & $(4.26)$ & $(3.44)$ & $(3.71)$ & $(3.98)$ & $(3.26)$ \\
Ira & 14.03 & 14.23 & 13.26 & 14.21 & 15.73 & 14.72 & 17.84 & 16.79 \\
& $(4.22)$ & $(4.60)$ & $(3.73)$ & $(4.22)$ & $(4.41)$ & $(4.31)$ & $(4.76)$ & $(4.81)$ \\
Hostilidad & 14.81 & 14.50 & 13.78 & 14.42 & 16.17 & 15.23 & 18.50 & 17.70 \\
& $(5.62)$ & $(5.37)$ & $(4.68)$ & $(5.63)$ & $(5.40)$ & $(4.69)$ & $(5.21)$ & $(5.62)$ \\
\hline
\end{tabular}




\section{Discusión y Conclusiones}

Los principales resultados de este estudio sugieren que la atribución de un estilo de socialización autoritario se relaciona con la manifestación de un comportamiento más agresivo y hostil hacia los iguales en comparación con la atribución de un estilo educativo democrático y permisivo. La percepción del empleo de una disciplina rígida se relaciona positivamente con las distintas formas de agresividad evaluada mientras que un patrón de relación negativa se obtiene cuando se considera la dimensión afecto. La percepción de elevados niveles de disciplina inductiva se asocia negativamente con los índices de agresividad física manifestados. Adicionalmente, los chicos recurrían con más frecuencia al empleo de formas físicas de agresión que las chicas.

Los hallazgos obtenidos ofrecen un apoyo parcial a las hipótesis establecidas dado que solo se observaron diferencias significativas entre la percepción del estilo de socialización democrático y negligente en el nivel de agresividad física informado. Asimismo, solo se alcanzaron diferencias estadísticamente significativas en función del sexo del participante en el nivel de agresividad física expresada, ya halladas en estudios previos (Bjorkqvist et al., 1992; Côté et al., 2007; Toldos, 2005; Underwood, Beron, Geutsch, Galperin y Risser, 2008). Sin embargo, las diferencias entre la percepción de un estilo de socialización democrático frente a otro autoritario aparecieron en todas las medidas consideradas.

Nuestros resultados son acordes a los de investigaciones precedentes que revelan que la percepción de un estilo parental autoritario mantiene relación con un incremento en la agresividad expresada. La apelación a la obediencia sin discusión, propia de un estilo autoritario, se vincula a la exhibición de conductas agresivas que podrían deteriorar seriamente el ajuste que los adolescentes exhiben en diferentes contextos como el de las interacciones con los iguales. Este hallazgo es consistente aún cuando una disciplina rígida no es reflejo de otras actuaciones más perniciosas como la manipulación, la inducción de culpa, la retirada de afecto o el control psicológico (Galambos, Barker y Almeida, 2003).

La relación observada entre el estilo de socialización y la agresividad, ira y hostilidad puede ser explicada por medio del modelado o reforzamiento vicario (Bandura, 1997). El aumento en la capacidad cognitiva permite atender, representar y posteriormente reproducir las conductas exhibidas por otros. Por tanto, niños y adolescentes adquieren mediante la ob- 
servación de modelos significativos, particularmente sus progenitores, que el ser hostil o agresivo puede reportar algún tipo de beneficio. Este hecho propicia que los chicos adopten estrategias agresivas en las interacciones que mantienen con sus iguales porque son comportamientos a los que están expuestos con relativa frecuencia (Kawabata et al., 2011).

Del mismo modo, los niños y adolescentes que reciben poco afecto y una imposición severa de normas pueden desarrollar un modelo mental de relaciones interpersonales caracterizado por la inseguridad e incertidumbre. Como resultado, pueden exhibir una falta de confianza tanto en sí mismos como en los demás que les sitúan en situación de riesgo en el ámbito social. Por tanto la rigidez y falta de sensibilidad que aprecian en sus hogares pueden incrementar la línea base de ira y hostilidad que se traduce en una mayor cantidad de emociones negativas y conductas agresivas en los intercambios con sus iguales (Underwood et al., 2008).

Por el contrario, la percepción de una maternidad y paternidad democrática se relacionó con el menor grado de expresión de conducta agresiva, ira y hostilidad. Menos concluyente es el beneficio asociado al estilo permisivo. A pesar de que las comparaciones a posteriori muestran un patrón de resultados prácticamente similar cuando el análisis se realiza en función del sexo del progenitor (beneficio asociado al ejercicio de un estilo permisivo), también parece demostrarse que la atribución de permisividad materna, especialmente por parte de los hijos varones, no se asocia con tan buenos resultados puesto que, en el caso particular de la agresividad verbal, los chicos que atribuyen a sus madres el desempeño de un estilo permisivo obtienen una puntuación más elevada en la dimensión señalada que los iguales que perciben a sus madres como negligentes y autoritarias. Una posible razón tal vez resida en que los hijos varones que perciben a sus madres como permisivas atribuyan a su otro progenitor un estilo educativo menos apropiado (negligente o autoritario), diluyéndose así el posible efecto benefactor que la maternidad permisiva conlleva en el caso de las chicas. No obstante, la percepción de congruencia o incongruencia en el ejercicio de la paternidad excede los objetivos planteados en este estudio.

Tal y como indica Steinberg (2001), los contextos familiares democráticos se relacionan con un mejor ajuste psicosocial en niños y adolescentes por tres razones. En primer lugar, el mayor afecto y aceptación hace que los hijos se muestren más receptivos a las influencias paternas. En segundo lugar, la adecuada combinación de implicación e imposición de límites 
al comportamiento de los hijos, propia del estilo democrático, facilita el desarrollo de habilidades de autorregulación que permiten a los jóvenes actuar de manera competente. Finalmente, la promoción del intercambio verbal entre padres e hijos hace a los últimos partícipes de un proceso que fomenta la competencia cognitiva y social, incrementando así su nivel de actuación más allá del marco familiar.

En esta línea, diversas investigaciones concluyen que la percepción por parte de los hijos adolescentes de unas influencias parentales positivas (afecto y calidad de los cuidados) así como una adecuada supervisión, actúan como elementos protectores que reducen la probabilidad de manifestación de conductas violentas y antisociales (Arm, Dahinten, Marshall y Shapka, 2011; Vieno, Nation, Pastore y Santinello, 2009). Concretamente, las relaciones afectivas eran promotoras de la autorrevelación de los hijos adolescentes que, a su vez, predecía un descenso en la expresión de la conducta agresiva hacia los iguales (Vieno et al., 2009). Así pues, los cuidados parentales pueden eliminar los sentimientos adolescentes de miedo, ira, inseguridad, emociones negativas que actúan como factores de riesgo para la aparición de problemas comportamentales.

El beneficio que una paternidad permisiva puede ocasionar en los hijos adolescentes, traducido en una menor expresión de agresividad, ira y hostilidad, ha sido señalado en otras investigaciones. En estudios recientes, García y Gracia $(2009,2010)$ obtuvieron que los hijos que definieron a sus padres como permisivos puntuaron igual que quienes los percibieron como democráticos y, aún mejor que aquellos otros que etiquetaron a sus padres como autoritarios y negligentes, en distintas medidas de ajuste psicosocial. Wolfradt, Hempel y Miles (2003) hallaron en su estudio con adolescentes alemanes que aquellos que percibían a sus padres como permisivos mostraban menores puntuaciones en despersonalización y ansiedad e informaban de un mayor empleo de estrategias activas para afrontar las situaciones estresantes.

Concluyendo, la expresión de altos niveles de conducta agresiva y hostil puede convertirse en un factor de riesgo asociado a la aparición de desajustes emocionales y comportamentales. Conocer los orígenes, uno de ellos las vivencias en el seno familiar, así como las consecuencias de la expresión del comportamiento agresivo, son elementos clave para la promoción y desarrollo de programas de prevención o intervención destinados a ayudar a los 
jóvenes a desarrollar un sentimiento de pertenencia al grupo y resolver conflictos de formas socialmente deseables (Underwood et al., 2009).

Este estudio presenta algunas limitaciones. La primera se relaciona con el empleo de cuestionarios o autoinformes para recabar la información de interés. Se presupone la honestidad de las respuestas, sin embargo, cabe la posibilidad de que los encuestados aporten información poco veraz con objeto de preservar una imagen positiva de sí mismos o de sus familias. En un intento de reducir las respuestas socialmente deseables se garantizó el anonimato de los participantes. En segundo lugar, el estudio tiene un carácter transversal de modo que no existe la posibilidad de probar hipótesis causales que exploren una posible direccionalidad en los resultados obtenidos. Finalmente, hemos de señalar que son los adolescentes los que actúan como únicos informantes, siendo más adecuada la obtención de información combinada (padres e hijos), o bien, la aportada por informadores independientes.

Los objetivos futuros residen en conocer los efectos que la congruencia o incongruencia percibida en el estilo de socialización de madres y padres tiene sobre diferentes medidas de ajuste adolescente. Algunos resultados de investigación (Hoeve et al., 2011; Fletcher, Steinberg y Sellers, 1999; McKinney y Renk, 2008; Simons y Conger, 2007) apuntan hacia un efecto paliativo de una paternidad incongruente cuando al menos uno de los progenitores emplea un estilo democrático. 


\section{Referencias}

Andreu, J. M., Peña, M. E. y Graña, J. L. (2002). Adaptación psicométrica de la versión española del Cuestionario de Agresión. Psicothema, 14, 476-482.

Arm, R. G., Dahinten, V.S., Marshall, S. K. y Shapka, J. D. (2011). An examination of the reciprocal relationships between adolescents' aggressive behaviors and their perceptions of parental nurturance. Journal of Youth Adolescence, 40, 207-220. doi:10.1007/s10964-009-9493-x

Armsden, G. C. y Greenberg, M. T. (1987). The Inventory of Parent and Peer Attachment: Individual differences and their relationship to psychological well-being in adolescence. Journal of Youth and Adolescence, 16, 427-454. doi:10.1007/BF02202939.

Bandura, A. (1997). Self-efficacy: The exercise of control. New York: W.H. Freeman.

Baumrind, D. (1971). Current theories of parental authority. Developmental Psychology Monographs, 4, 1-103. doi:10.1037/h0030372

Baumrind, D. (1991). The influence of parenting style on adolescent competence and substance use. Journal of Early Adolescence, 11, 56-95. doi:10.1177/02724316911110004.

Bersabé, R. M., Fuentes, M. J. y Motrico, E. (2001). Análisis psicométrico de dos escalas para evaluar estilos educativos parentales. Psicothema, 13, 678-684.

Bjorkqvist, K., Lagerspetz, K. M. y Kaukiainen, A. (1992). Do girls manipulate and boys fight? Developmental trends in regard to direct and indirect aggression. Aggressive Behavior, 18, 117-127. doi:10.1002/1098-2337

Buri, J. R. (1991). Parental Authority Questionnaire. Journal of Personality Assessment, 57, 110-119. doi:10.1207/s15327752jpa5701_13.

Buschgens, C., van Aken, M., Swinkels, S., Ormel, J., Verhulst, F., y Buitelaar, J. (2010). Externalizing behaviors in preadolescents: familial risk to externalizing behaviors and perceived parenting styles. European Child and Adolescence Psychiatry, 19, 567-575. doi:10.1007/s00787-009-0086-8.

Buss, A. H. y Perry, M. (1992). The Aggression Questionnaire. Journal of Personality and Social Psychology, 63, 452-459. doi:10.1037/0022-3514.63.3.452

Chao, R. K. (2001). Extending research on the consecuences of parenting style for Chinese Americans and European Americans. Child Development, 72, 1832-1843. doi:10.1111/1467-8624.00381.

Côté, S., Vaillancourt, T., Barker, E. D., Nagin, D. y Tremblay, R. E. (2007). The joint development of physical and indirect aggression: Predictors of continuity and change during 
childhood. Development and Psychopatology, 19, 37-55. doi:10.1017/S0954579407070034

Dwairy, M. A. (2008). Parental inconsistency versus parental authoritarianism: Associations with symptons of psychological disorders. Journal of Youth and Adolescence, 37, 616626. doi:10.1007/s10964-007-9169-3.

De la Torre, M. J., Casanova, P. F., García, M. C., Carpio, M. V. y Cerezo, M. T. (2011). Estilos educativos paternos y estrés en estudiantes de educación secundaria obligatoria. Psicología Conductual, 19, 577-590.

Estévez, E., Musitu, G. y Herrero, J. (2005). The influence of violent behavior and victimization at school on psychological distress: the role of parents and teachers. Adolescence, 40, 183-196.

Fletcher, A. C., Steinberg, L. y Sellers, E. B. (1999). Adolescents'well-being as a function of perceived interparental-consistency. Journal of Marriage and the Family, 61, 599-610.

Fuentes, M. J., Motrico, E. y Bersabé, R. M. (1999). Escala de Afecto (EA) y Escala de Normas y Exigencias (ENE): Versión hijos y versión padres. Universidad de Málaga.

Galambos, N. L., Barker, E. T. y Almeida, D. M. (2003). Parents do matter: Trajectories of change in externalizing and internalizing problems in early adolescence. Child Development, 74, 578-594. doi:10.1111/1467-8624.7402017

Gallarin, M. y Alonso-Arbiol, I. (2012). Parenting practices, parental attachment and aggressiveness in adolescence: A predictive model. Journal of Adolescence, 35, 1601-1610. doi:10.1016/j.adolescence.2012.07.002.

García, F. y Gracia, E. (2009). Is always authoritative the optimun parenting style? Evidence from Spanish. Adolescence, 44, 101-131.

García, F. y Gracia, E. (2010). ¿Qué estilo de socialización parental es el idóneo en España? Un estudio con niños y adolescentes de 10 a 14 años. Infancia y Aprendizaje, 33, 365384.

García-León, A., Reyes, G. A., Vila, J., Pérez, N., Robles, H. y Ramos, M. M. (2002). The Aggression Questionnaire: A validation study in students samples. The Spanish Journal of Psychology, 5, 45-53.

Glasgow, K. L., Dornbusch, S. M., Troyer, L., Steinberg, L. \& Ritter, P. L. (1997). Parenting styles, adolescents' attributions, and educational outcomes in nine heterogeneous high schools. Child Development, 68, 507-529. doi:10.1111/j.1467-8624.1997.tb01955.x

Hale III, W. W., van der Valk, I., Engels, R. \& Meeus, W. (2005). Does perceived parental rejection make adolescents sad and mad? The association of perceived parental rejec- 
tion with adolescent depression and aggression. Journal of Adolescent Health, 36, 466-474. doi:10.1016/j.jadohealth.2004.04.007

Hernando, A., Oliva, A. y Pertegal, M. A. (2012). Variables familiares y rendimiento académico en la adolescencia. Estudios de Psicología, 33, 51-65. doi:10.1174/021093912799803791

Hoeve, M., Dubas, J. S., Gerris, J. R. M., van der Laan, P. H. y Smeenk, W. (2011). Maternal and paternal parenting styles: Unique and combined links to adolescent and early adult delinquency. Journal of Adolescence, 34, 813-827. doi:10.1016/j.adolescence.2011.02.004

Kawabata, Y., Alink, L. R. A., Tseng, W., van IJzendoorn, M. H. y Crick, N.R. (2011). Maternal and paternal parenting styles associated with relational aggression in children and adolescents: A conceptual analysis and meta-analytic review. Developmental Review, 31, 240-278. doi:10.1016/j.dr.2011.08.001

Kim, S., Brody, G. H. y Murry, V. M. (2003). Longitudinal links between contextual risks, parenting, and youth outcomes in rural African American families. Journal of Black Psychology, 29, 359-377. doi:10.1016/S0140-1971(03)00059-9

Kistner, J., Counts-Allan, C., Dunke, S., Drew, C. H., David-Ferdon, C. y Lopez, C. (2010). Sex differences in relational and overt aggression in the late elementary school years. Aggressive Behavior, 36, 282-291. doi:10.1002/ab.20350

Lamborn, S. D. y Felbab, A. J. (2003). Applying ethnic equivalence and cultural values models to African American teens' perceptions of parents. Journal of Adolescence, 26, 605-622. doi:10.1177/0192513X06289649

Lamborn, S., Mounts, N., Steinberg, L. y Dornbusch, S. (1991). Patterns of competence and adjustment among adolescents from authoritative, authoritarian, indulgent, and neglectful homes. Child Development, 62, 1049-1065. doi:10.1111/j.14678624.1991.tb01588.x

Maccoby, E. E. y Martin, J. A. (1983). Socialization in the context of the family: Parent-child interaction. En P. H. Mussen (dir.), Handbook of child psychology, vol. 4 (pp. 1 -101). Nueva York: Wiley.

McKinney, C. y Renk, K. (2008). Differential parenting between mothers and fathers. Implications for late adolescents. Journal of Family Issues, 29, 806-827. doi:10.1177/0192513X07311222 
Morales-Vives, F., Codorniu-Raga, M. J. y Vigil-Colet, A. (2005). Características psicométricas de las versiones reducidas del cuestionario de agresividad de Buss y Perry. Psicothema, 17, 96-100.

Oliva, A., Parra, A. y Arranz, E. (2008). Estilos relacionales parentales y ajuste adolescente. Infancia y Aprendizaje, 31, 93-106. doi:10.1174/021037008783487093

Rodríguez, M. C., Peña, J. V. y Torío, S. (2009). La experiencia de la paternidad y la maternidad: análisis del discurso de las creencias sobre la crianza y el cuidado infantil. Infancia y Aprendizaje, 32, 81-95. doi:10.1174/021037009787138248

Santisteban, C. y Alvarado, J. M. (2009). The Aggression Questionnaire for Spanish preadolescents and adolescents. AQ-PA. The Spanish Journal of Psychology, 12, 320-326.

Simons, L. G., y Conger, R. D. (2007). Linking Mother-Father differences in parenting to a typology of family parenting styles and adolescent outcomes. Journal of Family Issues, 28, 211-245. doi: 10.1177/0192513X06294593.

Steinberg, L. (2001). We know some things: Parent-adolescent relationships in retrospect and prospect. Journal of Research on Adolescence, 11, 1-19. doi:10.1111/15327795.00001

Steinberg, L., Lamborn, S., Darling, N., Mounts, N. y Dornbusch, S. (1994). Over-time changes in adjustment and competence among adolescents from authoritative, authoritarian, indulgent, and neglectful families. Child Development, 65, 754-770. doi:10.1111/j.1467-8624.1994.tb00781.x

Toldos, M. P. (2005). Sex an age differences in self-estimated physical, verbal and indirect aggression in Spanish adolescents. Aggressive Behavior, 31, 13-23. doi:10.1002/ab.20034

Tur-Porcar, A., Mestre, V., Samper, P. y Malonda, E. (2012). Crianza y agresividad de los menores: ¿es diferente la influencia del padre y de la madre? Psicothema, 24, 284-288.

Underwood, M. K., Beron, K. J., Gentsch, J. K., Galperin, M. B. y Risser, S. D. (2008). Family correlates of children's social and physical aggression with peers: Negative interparental conflict strategies and parenting styles. International Journal of Behavioral Development, 32, 549-562. doi:10.1177/0165025408097134

Underwood, M. K., Beron, K. J. y Rosen, L. H. (2009). Continuity and change in social and physical aggression from middle childhood through early adolescence. Aggressive Behavior, 35, 357-375. doi:10.1002/ab.20313

Vaillancourt, T., Miller, J. L., Fagbemi, J., Côté, S. y Tremblay, R. E. (2007). Trajectories and predictors of indirect aggression: Results from a nationally representative longitudinal 
study of Canadian children aged 2-10. Aggressive Behavior, 33, 314-326. doi:10.1002/ab.20202

Vieno, A., Nation, M., Pastore, M. y Santinello, M. (2009). Parenting and antisocial behavior: A model of the relationship between adolescent self-disclosure, parental closeness, parental control, and adolescent antisocial behavior. Developmental Psychology, 45, 1509-1519. doi:10.1037/a0016929

Wolfradt, U., Hempel, S. y Miles, J. N. V. (2003). Perceived parenting styles, depersonalization, anxiety and coping behavior in adolescents. Personality and Individual Differences, 34, 521-532. doi:10.1016/S0191-8869(02)00092-2 